\title{
An artery accompanying the sciatic nerve (arteria comitans nervi ischiadici) and the position of the hip joint: a comparative histological study using chick, mouse, and human foetal specimens
}

\author{
A. Ishizawa ${ }^{1}$, S. Hayashi² ${ }^{2}$ H. Nasu ${ }^{3}$, H. Abe ${ }^{1}$, J.F. Rodríguez-Vázquez ${ }^{4}$, G. Murakami ${ }^{5}$ \\ ${ }^{1}$ Department of Anatomy, Akita University School of Medicine, Akita, Japan \\ ${ }^{2}$ Medical Education Center, Aichi Medical University, Aichi, Japan \\ ${ }^{3}$ Department of Anatomy, Tokyo Medical and Dental University School of Medicine, Tokyo, Japan \\ ${ }^{4}$ Department of Anatomy and Embryology II, Faculty of Medicine, Complutense University, Madrid, Spain \\ ${ }^{5}$ Division of Internal Medicine, Iwamizawa Kojin-kai Hospital, Hokkaido, Japan
}

[Received 16 October 2012; Accepted 12 November 2012]

\begin{abstract}
Birds and reptiles always carry a long and thick artery accompanying the sciatic nerve (i.e. the sciatic artery) whereas mammals do not. We attempted to demonstrate a difference in courses of the nerve and the arteries of foetuses in relation to hip joint posture. Eight mid-term human foetuses (15-18 weeks), five mouse foetuses (E18), and five chick embryos (11 days after incubation) were examined histologically. Thin feeding arteries in the sciatic nerve were consistently observed in human foetuses in spite of the long, inferiorly curved course of the nerve around the ischium. The tissue around the human sciatic nerve was not so tight because of the medial and inferior shift of the nerve away from the hip joint. The foetal hip joint position differed among the species, being highly flexed in humans and almost at right angle flexion in mice and chicks. Because of deep adduction of the hip joint in the mouse, the knee was located near the midline of the body. The mouse sciatic nerve ran through the tight tissue along the head of the femur, whereas the chick nerve ran through the loose space even in the gluteal region. In birds, evolution of the pelvis including the hip joint without adduction seemed to make the arterial development possible. In mammals, highly flexed or adducted hip joint seemed to be one of the disturbing factors against development of the long and thick artery. A slight change in posture may cause significant arterial variation. (Folia Morphol 2013; 72, 1: 41-50)
\end{abstract}

Key words: sciatic nerve, sciatic artery, hip joint, flexion, adduction, sirenomelia, human foetus

\section{INTRODUCTION}

In human mid-term foetuses, it is well known that the thigh is highly flexed at the hip joint (Fig. 1). This seems to lengthen the course of the sciatic nerve from the sacrum to the knee along the ex- tended (i.e. posterior or inferior) side of the hip. Despite this suggested lengthening of the nerve course in foetuses, many dissection studies have indicated the presence of an artery accompanying the adult sciatic nerve (arteria comitans nervi ischiadici) 


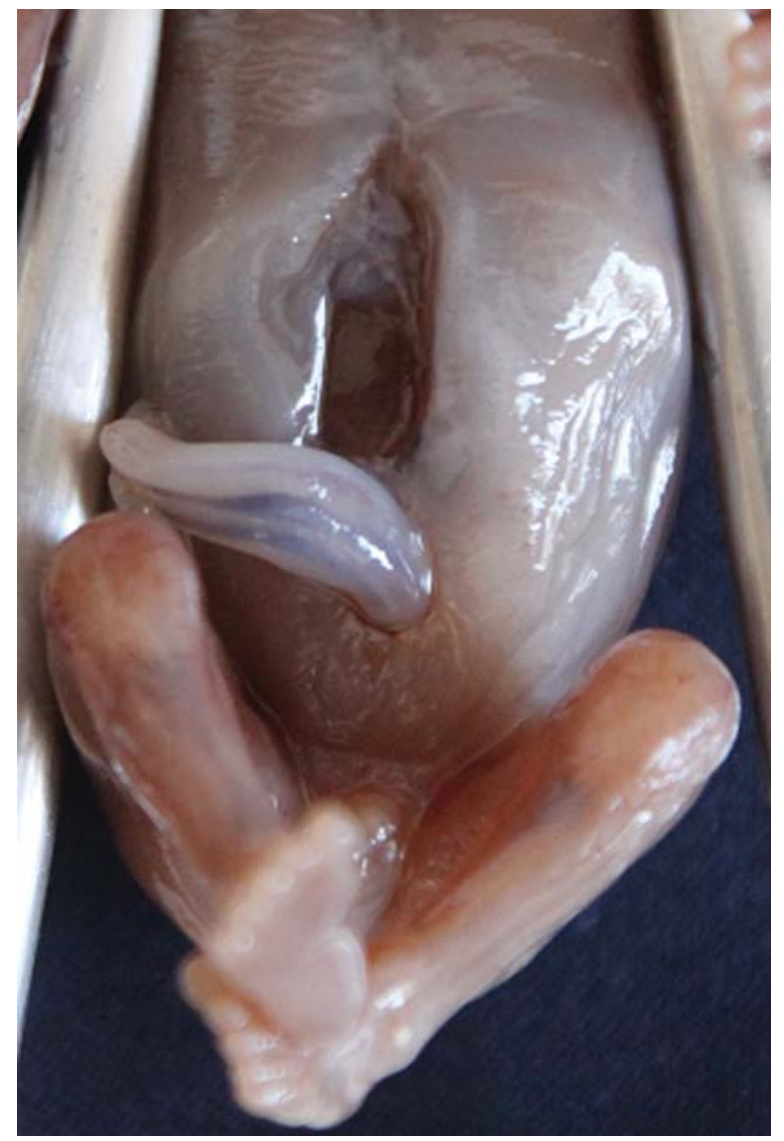

Figure 1. Anterior view of a human foetus at approximately 15 weeks of gestation. Because of the deep flexion position at the hip joint, the knee is located in the anterolateral side of the lower abdomen. Common abbreviation for figures: $A B$ - adductor brevis muscle; $\mathrm{AL}$ - adductor longus muscle; $\mathrm{AM}$ — adductor magnus muscle; $\mathrm{BF}$ - biceps femoris muscle; DFA — deep femoral artery; EIA — external iliac artery; FA — femoral artery; $\mathrm{FN}$ - femoral nerve; GE — gemellus muscles; $\mathrm{GM}$ - gluteus medius muscle; GMX - gluteus maximus muscle; GT - greater trochanter of the femur; IP — iliacus and psoas muscles; LCFA — lateral circumflex femoral artery; MCFA — medial circumflex femoral artery; $\mathrm{OE}$ - obturator externus muscle; $\mathrm{OI}$ - obturator internus muscle; $\mathrm{QF}$ - quadriceps femoris muscle; SM semimembranosus muscle; $\mathrm{SN}$ — sciatic nerve; $\mathrm{ST}$ — semitendinosus muscle; UA — umbilical artery.

$[1,5,7,8,14,15,21,33,36,37,40]$. Moreover, vascular surgeons have recently drawn attention to aneurysms of this accompanying artery as a cause of leg pain or paralysis, such reports $[6,18,22,25,34]$ having increased significantly in number since the year 2000. Kawashima and Sasaki [17] considered that most of these reported arteries were not a true remnant of the primitive sciatic artery, but rather a secondarily developed collateral branch, because of its course running posterior to the sciatic nerve. Does the foetal sciatic nerve have an accompanying space that would favour secondary development of a lon- gitudinal artery? The sciatic nerve receives multiple feeding arteries, especially from the deep femoral artery, in foetuses [38] as well as in adults [10, 16]. Consequently, the primary aim of this study was to determine the human foetal topographical anatomy of the sciatic nerve around the highly flexed hip joint.

In contrast to humans, the hip joint of birds and reptiles takes a neutral position for flexion-extension movement, and the thigh protrudes anteriorly or laterally [3, 32]. Thus, the sciatic artery as well as the femoral artery may be able to take a short-cut course from the pelvis to the knee without running along the extended aspect. According to Kawashima and Sasaki [17], unlike the longitudinal artery accompanying the human sciatic nerve (the so-called persistent sciatic artery; see the paragraph above), the sciatic artery in birds and reptiles runs along the anterior side of the sciatic nerve. People may consider that the difference in the arterial origin connects with that in the arterial course. However, we hypothesised that the hip joint position in foetuses is likely to be another critical factor determining the arterial anatomy because the joint position is responsible for whether the tissue along the sciatic nerve is tight or loose. To our knowledge, however, few previous studies of hip joint morphology have focused on its relationship to accompanying nerves and arteries. Consequently, the secondary aim of this study was to compare the foetal topographical anatomy of the hip area among the chick, mouse, and human. We chose the midterm foetuses for human materials because the hip joint is highly flexed: we tried to adjust the stages of hip joint development in the chick and mice materials.

\section{MATERIALS AND METHODS}

The study was performed in accordance with the provisions of the Declaration of Helsinki 1995 (as revised in Edinburgh 2000). We examined: 8 midterm human foetuses, comprising 3 foetuses at approximately 15 weeks of gestation ( 2 males and 1 female; crown rump length (CRL) 115-120 mm) and 5 foetuses at approximately 18 weeks of gestation ( 3 males and 2 females; CRL 150-160 mm); 5 mouse foetuses of 18 days of gestation (E18; ICR mouse); and 5 chick embryos at 11 days of incubation. The mouse foetuses were taken from a dam obtained from a local breeder, while fertilised eggs were also obtained from another breeder. The dam was sacrificed by deep anaesthesia whereas the animal foetuses or embryos were sacrificed by cutting of the neck. The specimens obtained were fixed with $10 \%$ 
$\mathrm{w} / \mathrm{w}$ formalin solution and stocked in $70 \% \mathrm{w} / \mathrm{w}$ ethanol solution.

All of the human specimens were part of the large collection kept at the Embryology Institute of the Universidad Complutense, Madrid, being the products of urgent abortion, miscarriages, or ectopic pregnancies managed at the Department of Obstetrics of the University. The donated human foetuses had been fixed in $10 \% \mathrm{w} / \mathrm{w}$ formalin solution and stocked in the same solution for 3-12 months. After trimming of the tissue mass including the pelvis, thigh, and knee, the specimens were decalcified in EDTA solution at $4{ }^{\circ} \mathrm{C}$ for 3 days $(0.5 \mathrm{~mol} / \mathrm{L}, \mathrm{pH} 7.5$; Decalcifying solution B, Wako, Tokyo). Approval for the study was granted by the ethics committee of the University.

To identify thin feeding arteries of the sciatic nerve, we observed horizontal histological sections of the 5 human mid-term foetuses at approximately 18 weeks. The sections were prepared semi-serially (5 microns thick; 100 -micron intervals). Most of the sections were stained with haematoxylin and eosin (HE staining), while some (2-3 sections per $1 \mathrm{~mm}$ thickness) were stained immunohistochemically to identify vessels or nerves. The primary antibodies used were 1) polyclonal anti-human S100 protein (Dako Z0311; Dako, Glostrup, Denmark) and 2) monoclonal anti-human alpha-1 smooth muscle actin (Dako M85; Dako, Glostrup, Denmark), and the second antibody reaction was detected via an HRP-catalysed reaction with diaminobenzidine. Hayashi et al. [12] and Miyake et al. [23] have reported that Dako M85 antibody stains the endothelium of foetal arteries and veins as well as any smooth muscle, but does not react with lymphatic endothelium. According to our experiences, for foetal arteries and veins, the antibody is better than well-known markers such as CD31 and von Willdebrand factor [31]. Counterstaining was performed using haematoxylin.

For comparative anatomical study of the nerves and vessels around the hip joint, we observed sagittal histological sections of: 3 human foetuses at approximately 15 weeks of gestation; 5 E18 mouse foetuses; and 5 chick embryos at 11 days of incubation. The sagittal sections seemed to be better than the horizontal or frontal sections for comparison of topographical anatomy of the nerve and artery around the hip joint. We chose these developmental stages for the three species because it was anticipated that the bones and muscles around the hip joint would be in similar states of development
$[26,29,39]$. After embedding in paraffin, sections 10 microns thick were cut at intervals of 50 microns. All sections were stained with $\mathrm{HE}$.

\section{RESULTS}

Feeding arteries of the foetal sciatic nerve: observations of human foetuses at 18 weeks

Immunohistochemistry using Dako M0851 antibody clearly demonstrated the endothelium of thin arteries (Figs. 2, 3). The vein was stained much more weakly than the artery. The highly flexed hip joint accompanied the sciatic nerve taking a curved course: initially it ran inferiorly (Fig. 2A, B), then turned anteriorly (Fig. 2C), and finally ran superiorly (Fig. 2A, B). Thus, the part of the sciatic nerve relatively distal to the gluteal region was seen "anterior" to the femoral nerve or the external iliac artery upon their arrival at the thigh. Notably, a single horizontal section demonstrated the deep femoral artery and its branch approaching the sciatic nerve (Fig. 3A). The gemellus muscles and the tendon of the obturator internus muscle were consistently interposed between the sciatic nerve and the ischium (Fig. 2C). However, the ischiadic tuberosity was located distantly from the course of the sciatic nerve (Fig. 2B, C).

In all 5 foetuses, despite its curved course around the ischium, the sciatic nerve consistently contained multiple thin arteries (Figs. 2D-F, 3). These arteries were branches of the inferior gluteal artery, the perforating artery, or the medial circumflexus femoral artery, according to observations of the near sections. Some of them supplied muscles around the nerve course (Fig. 2E). However, we did not find any long longitudinal artery along the nerve, possibly because of the fact that the semi-serial sections had been cut at 100-micron intervals. Although immunohistochemistry was not conducted, we did not find feeding arteries in the sciatic nerve in mouse and chick specimens used for comparison.

\section{Comparative foetal anatomy of the nerves and arteries around the hip joint}

Muscles of the thigh and gluteal region were well developed in all 3 species examined. In human foetuses at 15 weeks, spotty ossification was consistently evident in the head and neck of the femur, whereas ossification had already advanced in most of the ilium (Fig. 4). The ossification status in E18 mouse foetuses was similar to that in humans (Fig. 5). However, in chick embryos, ossification was consis- 

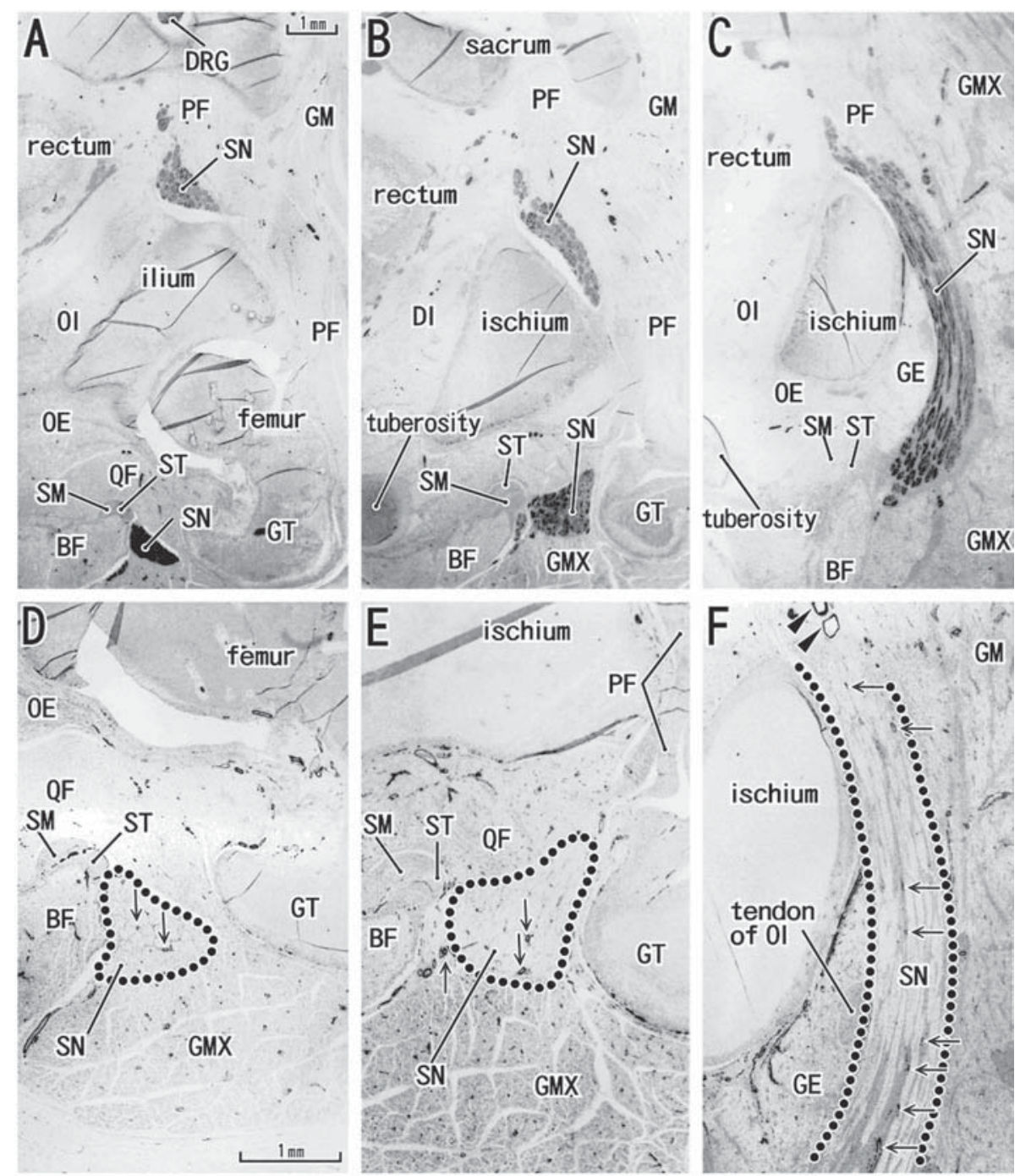

Figure 2. Sciatic nerve course around the ischium and thin arteries running in the nerve. A male human foetus at 18 weeks. Horizontal sections. Immunohistochemistry for nerves (panels A-C) or for vascular endothelium and smooth muscles (panels D-F). The lower side of the figure corresponds to the anterior side of the body. Panels $\mathbf{D}-\mathbf{F}$ are higher magnification views of the sciatic nerve in panels $\mathbf{A}-\mathbf{C}$, respectively. Panels A-C (or D-F) are prepared at the same magnification (scale bar in panel $\mathbf{A}$ or $\mathbf{D}$ ). Panel $\mathbf{A}$ (or $\mathbf{C}$ ) is the most superior (or inferior) in the figure. Intervals between panels are $1.0 \mathrm{~mm}(\mathbf{A}-\mathbf{B})$ and $1.2 \mathrm{~mm}(\mathbf{B}-\mathbf{C})$, respectively. Panel $\mathbf{A}$ includes both the intrapelvic part (posterior side of the body) and the relatively distal part (anterior side) of the sciatic nerve (SN). These two parts of the nerve are connected around the ischium in panel C. Panels D-F display thin arteries (arrows) running in the sciatic nerve. One of them (arrow with upper direction in panel $\mathbf{E}$ ) appears to supply the biceps femoris and gluteus maximus muscles (BF, GMX). Arrowheads in panel $F$ indicate branches of the inferior gluteal artery. A long accompanying artery of the sciatic nerve is not evident in this specimen; DRG — dorsal root ganglion; PF — piriformis muscle. Other abbreviations: see the common abbreviation — Fig. 1.

tently restricted to a limited part of the femoral shaft (Fig. 6). In human foetuses, the head of the femur was almost $2 \mathrm{~mm}$ in diameter whereas that in the mouse and chick ranged from 0.2 to $0.3 \mathrm{~mm}$. Thus, the bone head in human foetuses was almost 10 times larger than that in the mouse or chick. Likewise, the human femur was 6-7 times longer than its counterpart in mouse or chick (Table 1). Nevertheless, among the 3 species, the sciatic and femoral nerve roots showed a similar topographical relationship with the hip joint: the femoral nerve came from the far superior side of the hip joint, while the sciatic nerve originated at the level almost the same as the joint.

In human foetuses, the long shaft of the femur was always difficult to demonstrate in sagittal sections because it protruded superiorly, anteriorly, and laterally. Thus, to show the hip joint, the knee and most parts of the femur were removed from the lateral side of the paraffin block. The knee was located 18-23 mm superior and anterior to the ischium. Moreover, because of the slight abduction of the 


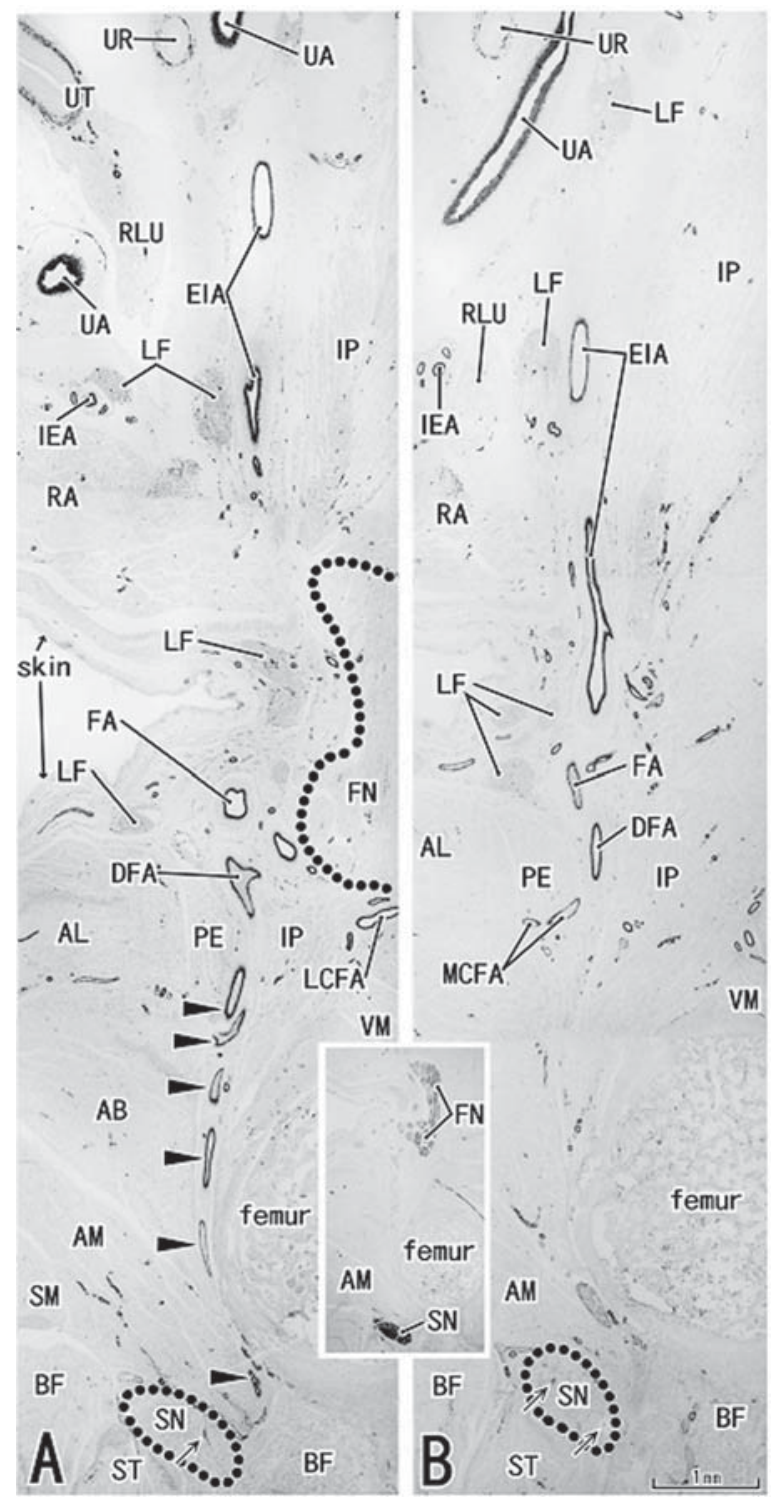

Figure 3. Horizontal section showing the deep femoral artery toward the sciatic nerve. A female human foetus at 18 weeks. Immunohistochemistry for vascular endothelium and smooth muscles. Panel $\mathbf{A}$ is located $0.6 \mathrm{~mm}$ superior to panel $\mathbf{B}$ and both panels are prepared at the same magnification (scale bar in panel B). The lower side of the figure corresponds to the anterior side of the body. An insert between these panels displays a lower half of panel $\mathbf{A}$ with immunohistochemistry for nerves. Because of the deep flexion position at the hip joint, the relatively distal part of the sciatic nerve (SN) is seen in the "anterior" side of the femoral nerve (FN in panel A) or the external iliac artery (ElA in panels A and B). Notably, a single horizontal section (panel A) demonstrates the first perforating artery (arrowheads) approaching the sciatic nerve. The sciatic nerve contains thin arteries (arrows in panels A and B); IEA — inferior epigastric artery; LF — lymph follicle; RA — rectus abdominis muscle; RLU — round ligament of the uterus; $\mathrm{PE}$ pectineus muscle; UR — ureter; UT — uterus; VM — vastus medialis muscle. Other abbreviations: see the common abbreviation — Fig. 1.

hip due to the thick thigh muscle masses, the knee was located 4-5 $\mathrm{mm}$ lateral to the ischium (Fig. 1). The femoral nerve arrived at the thigh lateral to the
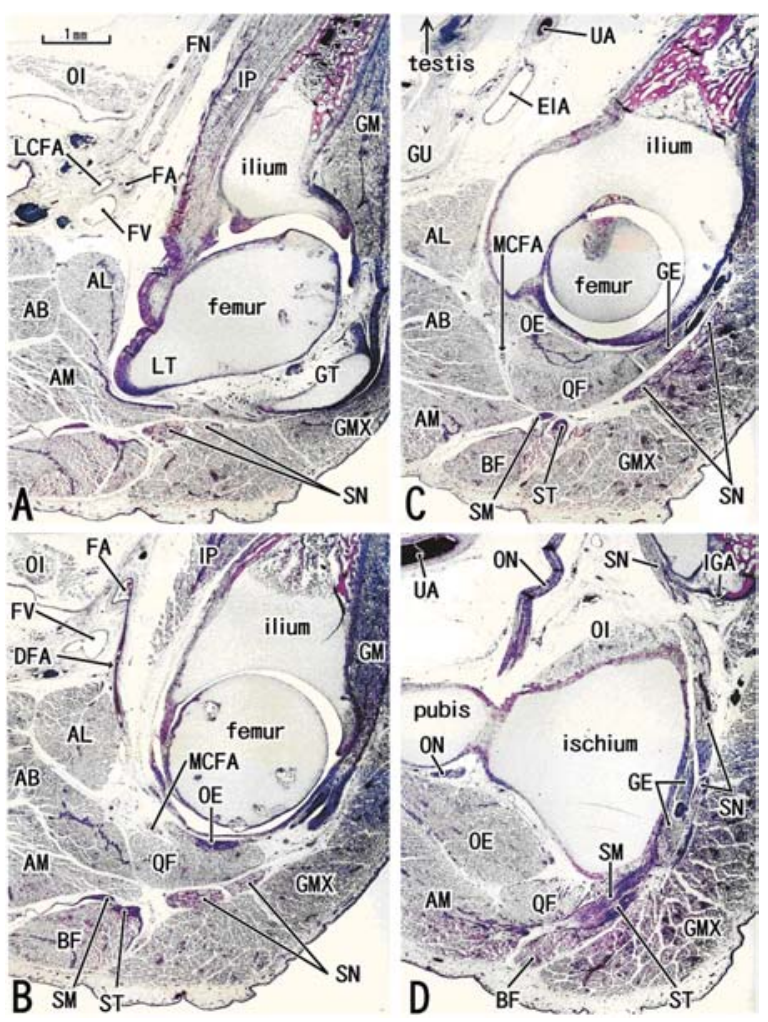

Figure 4. Human sciatic nerve running around the ischium in the medial side of the femoral nerve and artery arriving at the thigh. A male human foetus at 15 weeks. Sagittal sections. HE staining. The lower side of the figure corresponds to the inferior side of the body. All panels are prepared at the same magnification (scale bar in panel $\mathbf{A}$ ). Panel $\mathbf{A}$ (or $\mathbf{D}$ ) is the most lateral (or medial) in the figure. Intervals between panels are $1.2 \mathrm{~mm}(\mathbf{A}-\mathbf{B}, \mathbf{B}-\mathbf{C})$ and $1.6 \mathrm{~mm}$ (C-D), respectively. Ossification starts in the head and neck of the femur (panels A-C). Panel A includes both the femoral nerve and artery (FN, FA) arriving at the thigh and the relatively distal part of the sciatic nerve (SN). Panel B displays the deep femoral artery running inferiorly. Panel $\mathbf{D}$ exhibits the sciatic nerve running around the ischium; FV — femoral vein; GU — gubernaculums of the testis; IGA — inferior gluteal artery; LT — lesser trochanter of the femur; $\mathrm{OI}$ - obliquus internus muscle; $\mathrm{ON}$ - obturator nerve. Other abbreviations, see the common abbreviation — Fig. 1.

femoral head, whereas the sciatic nerve ran around the ischium medial to the head in 2 of the 3 foetuses examined (Fig. 4). In another specimen, the sciatic nerve exhibited a curved course inferior to the head of the femur. However, the gemellus muscles and the tendon of the obturator internus muscle were consistently interposed between the sciatic nerve and the bone. Thus, the sciatic nerve was not attached to the femoral head. The deep femoral artery appeared to run inferiorly in sagittal sections (Fig. 4B).

In mouse foetuses, the relatively short femur $(3.7-3.8 \mathrm{~mm})$ protruded anteromedially. The knee was located almost $3 \mathrm{~mm}$ medial and $2 \mathrm{~mm}$ anterior to the hip joint. Thus, considerable adduction of 

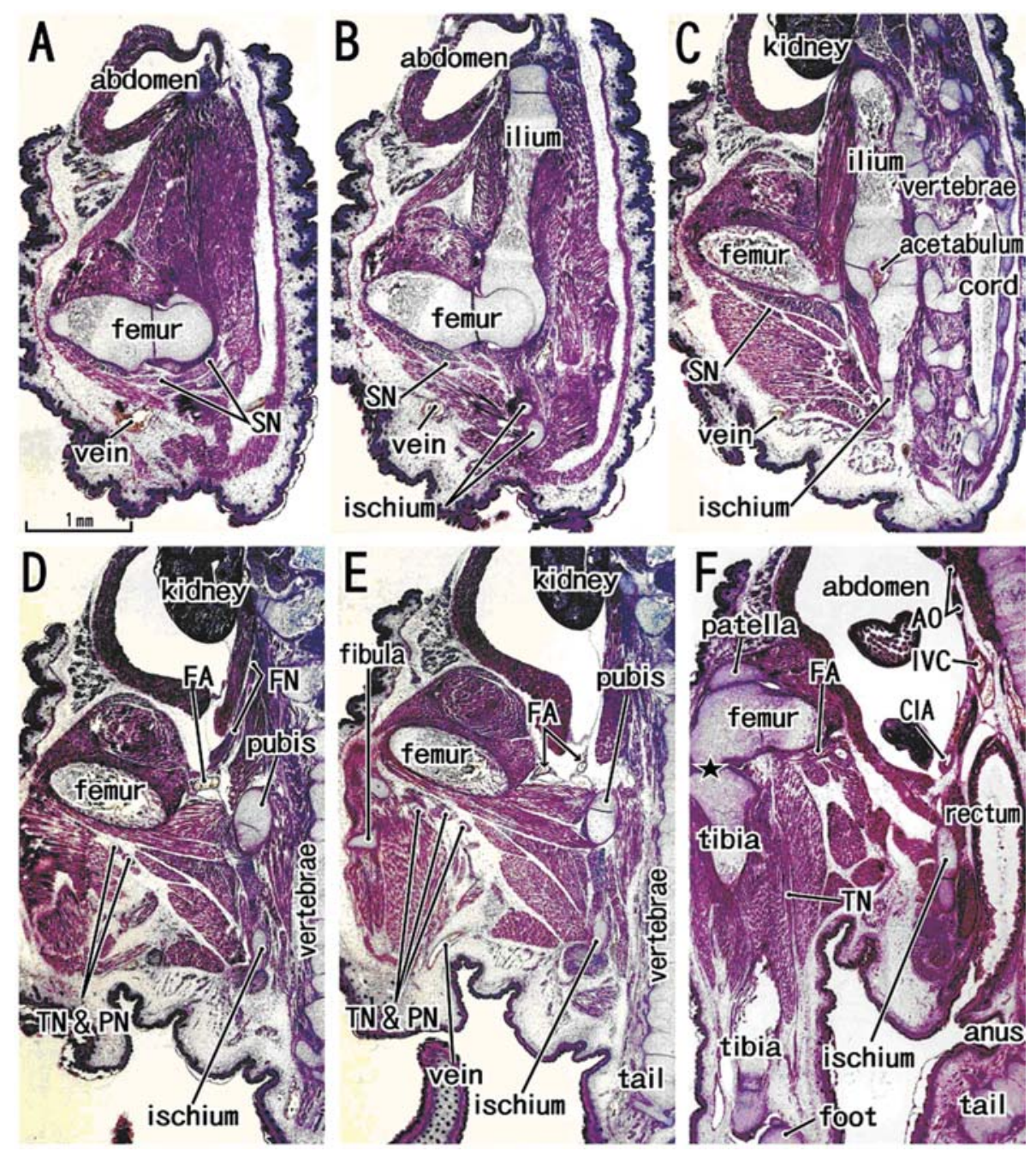

Figure 5. Mouse sciatic nerve running along the head of the femur in the lateral side of the femoral nerve and artery arriving at the thigh. E18 mouse foetus. Sagittal sections. HE staining. The lower side of the figure corresponds to the inferior side of the body. All panels are prepared at the same magnification (scale bar in panel $\mathbf{A}$ ). Panel $\mathbf{A}$ (or $\mathbf{F}$ ) is the most lateral (or medial) in the figure. Intervals between panels are $0.4 \mathrm{~mm}(\mathbf{A}-\mathbf{B}), 1.1 \mathrm{~mm}(\mathbf{B}-\mathbf{C}), 0.8 \mathrm{~mm}(\mathbf{C}-\mathbf{D}), 0.3 \mathrm{~mm}(\mathbf{D}-\mathbf{E})$, and $0.9 \mathrm{~mm}(\mathbf{E}-\mathbf{F})$, respectively. Ossification is seen in the shaft of the femur and the ilium (panels A-E). Panel $\mathbf{A}$ includes the sciatic nerve (SN) running along the head of the femur. The ischium is away from the nerve course (panels B-E). Panels $\mathbf{D}$ and $\mathbf{E}$ display the femoral nerve and artery (FN, FA) arriving at the thigh. The knee joint (star in panel F) is seen with the anus in the almost mid-sagittal plane; AO — aorta; CIA — common iliac artery; IVC — inferior vena acva; $\mathrm{PN}$ — peroneal nerve; TN — tibial nerve. Other abbreviations, see the common abbreviation — Fig. 1.

the hip was evident relative to the length of the femur (Fig. 5). Subsequently, the knee was included in the same sagittal plane as the anus (Fig. 5F). In contrast to the human hip joint, there was no deep flexion, and the ilium was much longer than the femur. After passing through the large sciatic foramen, the sciatic nerve turned along the head of the femur from the inferior to the anterior side (Fig. 5A). At the most inferior point of its course, the sciatic nerve was in contact with the hip joint, and not to the ischium. We found no muscles interposing between the nerve and the bone. The femoral nerve and artery were located medial to the sciatic nerve course (Fig. 5D, E). In spite of the horizontal position of the femur, the extensor muscles of the thigh, including the iliacus and psoas muscles, were prominently developed and located closely to the abdominal wall muscles.

In 11-day chick embryos, the relatively long femur simply protruded anteriorly. The knee was located $4 \mathrm{~mm}$ anterior and $1 \mathrm{~mm}$ superior to the hip joint. Notably, therefore, the sciatic nerve, the sciatic artery, femoral artery and femoral nerve were seen together in the thigh within sagittal sections $0.1-$ $-0.2 \mathrm{~mm}$ thick (Fig. 6): this neurovascular zone was located $0.1 \mathrm{~mm}$ medial to the femoral shaft. More- 


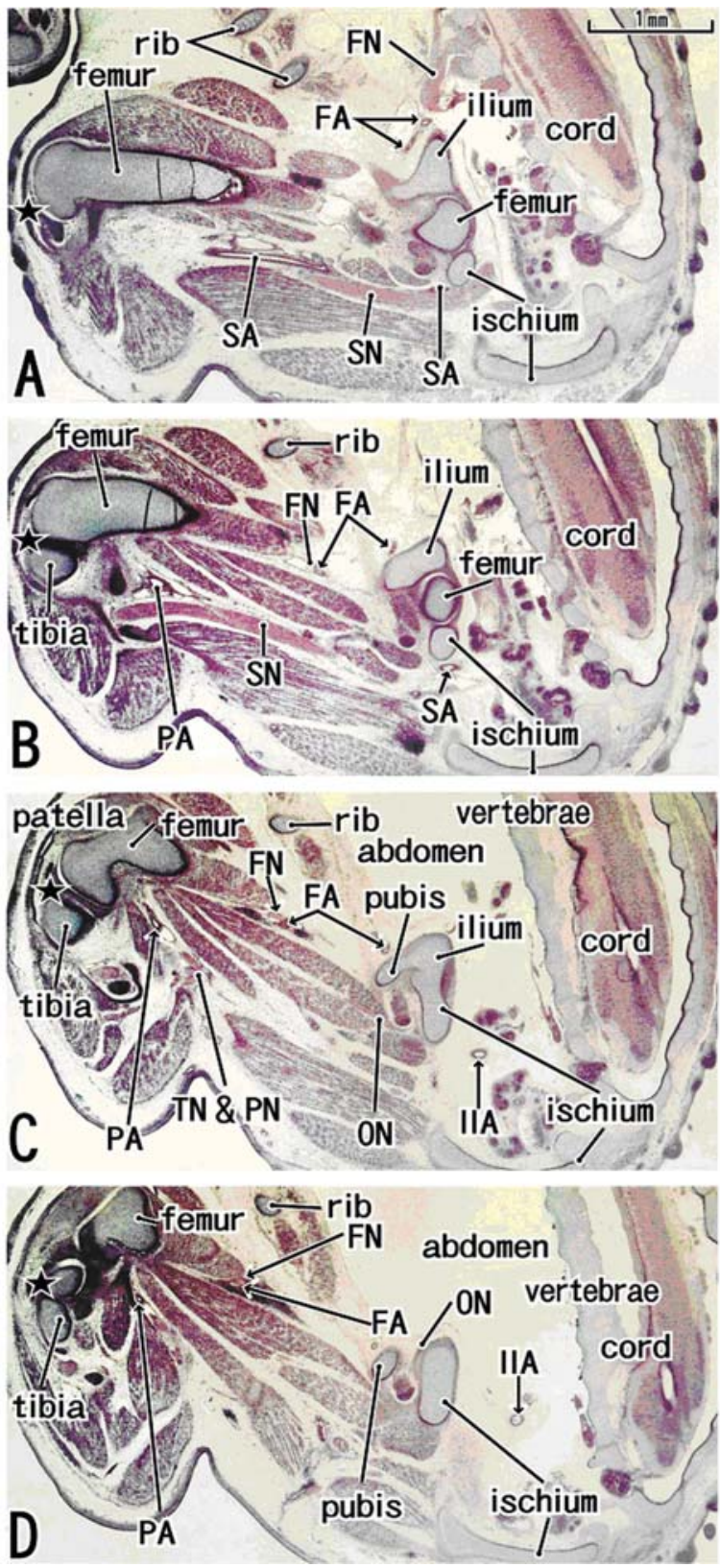

Figure 6. Chick sciatic nerve and artery seen in the same sagittal plane with the femoral nerve and artery. Chick embryo after 11 days' incubation. Sagittal sections. HE staining. The lower side of the figure corresponds to the inferior side of the body. All panels are prepared at the same magnification (scale bar in panel A). Panel A (or D) is the most lateral (or medial) in the figure. Intervals between panels are $0.1 \mathrm{~mm}$, respectively. Ossification is limited in the shaft of the femur (in the lateral side of panel A). Panel $\mathbf{A}$ includes the longitudinal courses of the sciatic nerve and artery (SN, SA) as well as the femoral nerve and artery (FN, FA) arriving at the thigh. The ischium is away from the sciatic nerve course (panels $\mathbf{A}-\mathbf{D}$ ). The knee joint (star) is seen in just the anterior side of the hip joint; IIA — internal iliac artery; $\mathrm{On}$ — obturator nerve; PA — popliteal artery; $\mathrm{PN}$ - peroneal nerve; TN — tibial nerve.

over, the knee and hip joints were visible in the same sagittal section: thus there was little or no medial or lateral shift of the knee. The thigh muscles were packed more loosely in the thigh than in human and mouse foetuses.

Consequently, the foetal hip joint position differed between species, showing 1) a highly flexed position in human foetuses and 2) almost right angle flexion in mouse foetuses and chick embryos. In addition, because the mouse hip joint showed deep adduction, the knee was located near the midline of the body. In mouse foetuses, the sciatic nerve ran through the tight tissue along the head of the femur, whereas in the chick the nerve ran through the loose space, even in the gluteal region. Although the human sciatic nerve took a long, inferiorly curved course around the ischium, the tissue around the nerve appeared to be not so tight because of the medial shift of the nerve away from the hip joint. Figure 7 and Table 1 summarise the details of 1) the sizes of the femur, 2) the position of the hip joint, 3 ) the course of the sciatic nerve, and 4) the course of the femoral artery. For the human specimen, the scale of the morphology in the figure is reduced to $1 / 4$ to match those of the mouse and chick.

\section{DISCUSSION}

The present study demonstrated a difference in the course of the foetal sciatic nerve relative to the hip joint among the human, mouse, and chick. Because the sciatic and femoral nerve roots showed a similar topographical relationship with the hip joint among the species, the difference in the sciatic nerve course seemed to depend on the species-specific position of the hip joint: the mouse hip joint was characterised by deep adduction, whereas the human hip joint was characterised by deep flexion. Moreover, the human sciatic nerve was able to pass medially to the joint, perhaps allowing a course through looser tissue than the lateral course in the mouse. The present study also demonstrated abundant thin feeding arteries in the human foetal sciatic nerve as a constant morphologic feature, in spite of the inferiorly curved course. The early development of the feeding arteries in humans seemed to depend on the medial and inferior shift of the nerve without mechanical tension from the head of the femur. Nevertheless, in the late stage in utero or during postnatal life, the tight tissue along the fetal mouse sciatic nerve around the head of the femur would likely change drastically to allow secondary development of a distinct accompanying artery: in fact, Bell and Weddell [4] have reported this to be a constant morphologic feature in the adult rat (see below). 
Table 1. Size and position of the foetal hip joint in specimens examined

\begin{tabular}{lcccc}
\hline & Femur head & Femur length & Knee to the femur head & Sciatic nerve course \\
\hline Chick & $0.2 \mathrm{~mm}$ & $4.1-4.2 \mathrm{~mm}$ & Anterior & Inferior to the femur head \\
Mouse & $0.3 \mathrm{~mm}$ & $3.7-3.8 \mathrm{~mm}$ & Anteromedial & Attaching to femur head \\
Human & $2 \mathrm{~mm}$ & $25-30 \mathrm{~mm}$ & Anterosuperior & Medio-inferior to the femur head \\
\hline
\end{tabular}

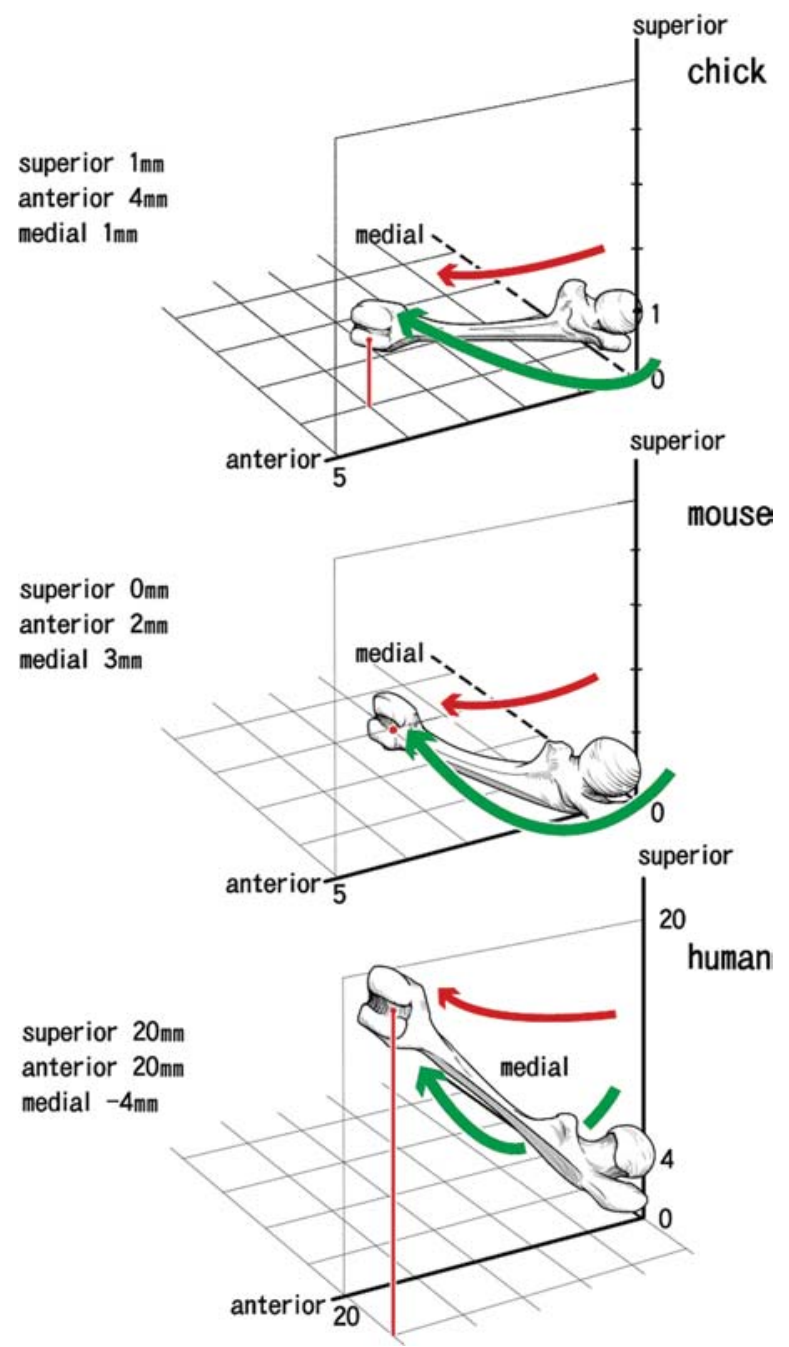

Figure 7. Summary of the comparative anatomy of the foetal hip joint position and sciatic nerve course. For simple representation, the adult human femur is drawn for foetuses of all 3 species. Green arrow (or red arrow) indicates the sciatic nerve course (or the femoral arterial course). The co-ordinates $(0=$ hip joint) are composed of the superior, anterior, and medial axes. The knee position in a typical specimen is shown by 3-dimensional positions $(\mathrm{mm})$ from the hip joint. The scale of the human morphology in the figure is reduced into $1 / 4$ to match those of the mouse and chick. The chick knee is located in the anterior side of the hip joint; the mouse knee is medial, rather than anterior, to the hip joint and; the human knee is anterosuperior and slightly lateral.

According to textbooks on human development $[2,24,30]$, the sciatic artery branching from the internal iliac artery is considered to be the primitive posterior axial artery in the lower limb bud, whereas the femoral artery branching from the external iliac artery is the anterior axial artery in the proximal part of the lower limb. Usually, the femoral artery replaces the main arterial trunk by joining the popliteal artery. The sciatic artery in birds and reptiles runs anteriorly to the sciatic nerve $[13,17]$. Although many reports have referred to the "persistent sciatic artery" (see Introduction), the sciatic artery proper seems to be very rare in adults (4 out of 1724 cadavers, $0.23 \%$ ) [20] when it is defined as running a course anterior to the sciatic nerve in the thigh. Conversely, however, the artery accompanying the sciatic nerve (arteria comitans nervi ischiadici) running posteriorly to the nerve may often be evident in human cadavers. Likewise, in adult rats, Bell and Weddell [4] described this artery as a regular morphologic feature. Thus, the mouse is also likely to carry such an artery. Among the three species examined in the present study, the human and mouse are likely to carry the accompanying artery, whereas the chick carries the sciatic artery proper.

It is noteworthy that variable development of the sciatic artery is normally evident in "sirenomelia", a rare limb malformation exhibiting a spectrum of fusion of the bilateral lower extremities, as well as by the single or impar umbilical artery of the high origin from the aorta $[9,35]$. The reported incidence of sirenomelia in humans ranges from 1.1 to 4.2 per 100,000 births (reviewed by Garrido-Allepuz et al. [9]). Sirenomelia occurs in mice lacking Cyp26a1, an enzyme that degrades retinoic acid, and also in mice that are partially deficient in bone morphogenic protein. However, sirenomelia is unaccompanied by abnormality of either the vertebral column or spinal nerves, in contrast to the caudal body defects for which mutation of the Hox gene seems to be responsible. Thus, the unusual sciatic artery in adult mammals is unlikely to be a result of failure or alteration in the basic body plan, but of simple redundancy of cell signalling at a limited site such as the dorsocau- 
dal organisation centre for the lower limb bud. In sirenomelia, it has been hypothesised that vascular steal may be responsible for one of the phenotypes due to abnormality of the umbilical artery. However, to our knowledge, none of the previous reports of a persistent sciatic artery took into account umbilical morphology. Conversely, even without any limb abnormality, a patent impar umbilical artery has been reported to accompany a severe pelvic arterial variation comprising 1) the usual femoral arteries, 2) highly developed gluteal arteries, and 3) unusual arterial communication in an 18-year-old woman [11].

Classically, Nakagawa et al. [27] have reported that subclasses of bHLH transcription factors contribute to the formation of both the aortic arch arteries and the limb buds. Recently, Zhao et al. [41] reported a gene that is responsible for both vascular anatomy and limb morphogenesis. Indeed, the major arteries in the trunk, such as the aorta and its primary divisions, may be determined by gene cascade(s). However, it seems possible that the mammalian sciatic artery in the early limb bud regresses due to a region-specific factor such as mechanically tight tissue or frequent mechanical stress from movement of the hip joint. Conversely, if the hip joint of the chick embryo is experimentally changed to a highly flexed and adducted position, would the sciatic artery stop developing and be replaced by the femoral artery? We consider this to be very likely. In other words, genomic determination of sciatic artery development, if present, seems to depend on a body plan to form the anteriorly protruding thigh in the absence of adduction. Thus, it is likely that, during evolution, determination of the pelvic morphology including the hip joint occurs first, and then arterial development in the thigh follows changes in the pelvis. In humans, a thick artery accompanying the sciatic nerve may replace the femoral artery when the foetal hip joint takes a "pathologically" neutral or extended position. Likewise, rare variations of the femoral, obturator, and inferior epigastric arteries (e.g. Nasu and Chiba [28]) may simply be due to a slight change in the posture of the foetal lower extremities. In addition, we have not been able to find any previous reference to whether or not a history of intrauterine thalidomide exposure causes the persistent sciatic artery to develop in mammals, although it causes anomalies of the cardiovascular system as well as the lower extremities (e.g. Kligerman et al. [19]).
Overall, rather than redundancy in the genomically defined body plan, a slight change in posture of the foetal lower extremities may cause significant arterial variation such as the persistent sciatic artery. A rare limb malformation sirenomelia, in which the sciatic artery is normally present, suggests close correlation between the posture of the lower extremities and the sciatic artery development.

\section{REFERENCES}

1. Adachi B (1928) Das Arteriensystem der Japaner. Band II, Kyoto Univ Press, Kyoto, pp. 136-142.

2. Arey LB (1954) Developmental Anatomy. A textbook and laboratory manual of embryology. $6^{\text {th }}$ Ed. Saunders, Philadelphia.

3. Baumel JJ, King AS, Lucas AM, Breazile JE, Evans HE (1979) Nomina Anatomica Avium. Brace Jovanovich Publication, London, pp. 202-206, 214-219.

4. Bell MA, Weddell AG (1984) A descriptive study of the blood vessels of the sciatic nerve in rat, man and other mammals. Brain, 107: 871-898.

5. Blair CB, Nandy K (1965) Persistence of the axis artery of the lower limb. Anat Rec, 152: 161-172.

6. Chleboun JO, Teasdale JE (1995) A pulsatile gluteal mass due to sciatic artery aneurysm. Aust N Z J Surg, 65: 907-910.

7. Finerty JC (1947) Persistent ischiatic artery. Anat Rec, 98: 587-595.

8. Gabrielli C, Olave E, Sarmento A, Mizusaki C, Prates JC (1997) Abnormal extrapelvic course of the inferior gluteal artery. Surg Radiol Anat, 19: 139-142.

9. Garrido-Allepuz C, Haro E, Gonález-Lamuńo D, Martínez-Frias ML, Bertocchini F, Ros MA (2011) A clinical and experimental overview of sirenomelia: insight into the mechanosims of congenital limb malformations. Dis Model Mech, 4: 289-299.

10. Georgakis E, Soames R (2008) Arterial supply to the sciatic nerve in the gluteal region. Clin Anat, 21: 62-65.

11. Glodny B, Henninger B, Hofmann $K$, Trieb T, Peterson J, Rehder P (2009) CT appearance of a patent impar umbilical artery in a adult woman and related anomalies: a case report and review of the literature. Cases J, 2: 65 .

12. Hayashi S, Murakami G, Ohtsuka A, Itoh M, Nakano T, Fukuzawa Y (2008) Connective tissue configuration in the human liver hilar region with special reference to the liver capsule and vascular sheath. J Hep Bil Pancr Surg, 15: 640-647.

13. Hoshino Y, Kawashima T, Miyazawa M, Sasaki H (2004) A comparative anatomical study of the sciatic artery. Morphol Sci, 8: 19-24.

14. Inoue K, Yamaai T, Odajima G (1993) Persistent aciatic artery: case report. Okajimas Folia Anat Jpn, 69: 311-314.

15. Job TT (1933) Persistence of a left ischiatic artery. Anat Rec, 58: 101-105.

16. Karmańska W, Mikusek J, Karmański A (1993) Nutrient arteries of the human sciatic nerve. Folia Morphol, 52: 209-215. 
17. Kawashima T, Sasaki H (2010) Reasonable classical concepts in human lower limb anatomy from the viewpoints of the primitive persistent sciatic artery and twisting human lower limb. Okajimas Folia Anat Jpn, 87: 141-149.

18. Kim HJ, Cho YS, Lim H (2008) Persistent sciatic artery with monoplegia in right lower leg without vascular complication symptoms in an obese woman. J Emerg Med, 34: 291-294.

19. Kligerman S, Blum A, Abbara S (2009) Persistent fifth aortic arch in a patient with a history of intrauterine thalidomide exposure. J Cardiovasc Comp Tomogr, 3: 412-414.

20. Kodama K (2000) Arterial system. In: Sato T, Akita K eds. Anatomic variations in Japanese. University of Tokyo Press, Tokyo, pp. 260-261.

21. Kubota K, Haibara T, Noguchi M (1957) A case of bilateral existence of ischiadic artery in man. Okajimas Folia Anat Jpn, 30: 339-340.

22. Mazet N, Soulier-Guerin K, Ruivard M, Garcier JM, Boyer L (2006) Bilateral persistent sciatic artery aneurysm discovered by atypical sciatica: a case report. Cardiovasc Intervent Radiol, 29: 1107-1110.

23. Miyake N, Hayashi S, Cho BH, Kawase T, Murakami G, Fujimiya M, Kitano H (2010) Fetal anatomy of the human carotid sheath and structures in and around it. Anat Rec, 293: 438-445.

24. Moore KL, Persaud TVN (2008) The developing human. Clinically oriented embryology. Chapter 16, The limbs. $8^{\text {th }}$ Ed. Saunders, Philadelphia.

25. Mousa A, Rapp Parker A, Emmett MK, AbuRhama A (2010) Endovascular treatment of symptomatic persistent sciatic artery aneurysm: a case report and review of literature. Vasc Endovascular Surg, 44: 312-314.

26. Murakami G, Nakamura H (1991) Somites and the pattern formation of trunk muscles: a study in quail-chick chimera. Arch Histol Cytol, 54: 249-258.

27. Nakagawa O, Nakagawa M, Richardson JA, Olson EN, Srivastava D (1999) HRT1, HRT2, and HRT3: a new subclass of bHLH transcription factors marking cardiac, somitic, and pharyngeal arch segments. Dev Biol, 216: 72-84.

28. Nasu H, Chiba S (2009) Rare case of femoral artery ramification and origin of the obturator artery. Anat Sci Int, 84: 323-326.
29. Niikura H, Jin ZW, Cho BH, Murakami G, Yaegashi N, Lee JK, Lee NH, Li CA (2010) Human fetal anatomy of the coccygeal attachments of the levator ani muscle. Clin Anat, 23: 566-574.

30. Patten BM (1968) Human Embryology. $3^{\text {rd }}$ Ed. Chapter 20. The circulatory system. McGraw-Hill, New York.

31. Pusztaszeri MP, Seelentag W, Bosman FT (2006) Immunohistochemical expression of endothelial markers CD31, CD34, von Willebrand factor, and Fli-1 in normal human tissues. J Histochem Cytochem, 54: 385-395.

32. Romer AS, Parsons TS (1977) The vertebrate body. $5^{\text {th }}$ Ed. Saunders, Philadelphia.

33. Sekiya S, Horiguchi M, Komatsu H, Kowada S, Yokoyama S, Yoshida K, Isogai S, Nakano M, Koizumi M (1997) Persistent primitive sciatic artery associated with other various anomalies of vessels. Acta Anat Nippon, 158: 143-149.

34. Shutze WP, Garrett WW, Smith BL (1993) Persistent sciatic artery: collective review and management. Ann Vasc Surg, 7: 303-310.

35. Talamo TS, Macpherson TA, Dominquez R (1982) Siremia. Amgiographic demonstration of vascular anomalies. Arch Pathol Lab Med, 106: 347-348.

36. Tohno Y, Tohno S, Watanabe T, Watanabe M, Kimura Y (1993) Anomaly of bilateral persistent sciatic arteries. Acta Anat Nippon, 68: 422-428.

37. Ukeshima A, Yoshimura R, Wake N, Watanabe T, Yamamoto M, Yoshioka Y, Yoshioka M, Yoshimoto T, Fujimoto T (1990) Bilateral persistent sciatic arteries. Okajimas Folia Anat Jpn, 67: 1-4.

38. Ugrenovic SZ, Jovanovic ID, Vasovic LP, Stefanovic BD (2007) Extraneural arterial blood vessles of human fetal sciatic nerve. Cells Tissues Organs, 186: 147-153.

39. Yamaguchi K, Kiyokawa J, Akita K (2008) Developmental process and ectodermal contribution to the anal canal in mice. Ann Anat, 190: 119-128.

40. Yoshimura R, Wake N, Watanabe T, Yamamoto M, Yoshioka Y, Yoshioka M, Yoshimoto T, Ukeshima A, Fujimoto T (1988) A case of bilateral sciatic artery. Acta Anat Nippon, 67: 83-99. In Japanese with English abstract

41. Zhao F, Bosserhoff AK, Burttner R, Moser M (2011) A hear-hand syndrome gene:Tfap $2 \mathrm{~b}$ plays a critical role in the development and remodeling of mouse ductus arteriosus and limb patterning. PLoS One, 6: e22908. 